\title{
Global Astrophysical Telescope System - GATS
}

\section{Polińska, K. Kamiński, W. Dimitrov, M. Fagas, W. Borczyk, T. Kwiatkowski, R. Baranowski, P. Bartczak, and A. Schwarzenberg-Czerny}

Astronomical Observatory Institute, Faculty of Physics, Adam Mickiewicz University, Słoneczna Street 36, 60-286 Poznań, Poland email: polinska@amu.edu.pl

\begin{abstract}
The Global Astronomical Telescope System is a project managed by the Astronomical Observatory Institute of Adam Mickiewicz University in Poznań (Poland) and it is primarily intended for stellar medium/high resolution spectroscopy. The system will be operating as a global network of robotic telescopes. The GATS consists of two telescopes: PST 1 in Poland (near Poznań) and PST 2 in the USA (Arizona). The GATS project is also intended to cooperate with the BRITE satellites and supplement their photometry with spectroscopic observations.
\end{abstract}

Keywords. instrumentation: spectrographs, techniques: radial velocities, spectroscopic, stars: binaries, oscillations (including pulsations).

The first telescope, PST 1 (Poznań Spectroscopic Telescopes), was designed as a spectroscope producing medium/high resolution spectra. The telescope has been in operation at Borowiec Astrogeodynamic Observatory near Poznań (Poland) since summer 2007, and its description can be found in Baranowski et al. (2009). The other telescope, PST 2 , is scheduled to start regular observations in the end of 2013 at a site in Arizona, USA (for more information see Kamiński et al., these proceedings).

The PST 1 consists of a twin 0.5-m Newtonian telescopes, fibre-fed echelle spectrograph with a resolution $R \sim 35000$, and a low-noise back-illuminated $2 \mathrm{k} \times 2 \mathrm{k}$ Andor DZ 436 CCD camera. The telescope is embedded on an equatorial, robotic mount and is capable of performing remotely-controlled observations. The PST 1 may be used in several different modes. In the basic mode only a single mirror is used for spectroscopy. In the second mode both mirrors are used to produce two simultaneous echelle spectra of the same object. Such parallel spectra can be used to increase signal-to-noise $(\mathrm{S} / \mathrm{N})$ ratio or help to remove cosmic rays. The third mode allows for simultaneous photometric and spectroscopic observations.

Results of radial velocity measurements done so far with the PST 1 show a stability at a level of $100 \mathrm{~m} \mathrm{~s}^{-1}$. This corresponds to about $1 / 25$ of the pixel size of our CCD $(13.5 \mu \mathrm{m})$. Thanks to its light-efficient design and low-noise camera, we are able to achieve $\mathrm{S} / \mathrm{N} \sim 100$ spectrum for a $V=7 \mathrm{mag}$ star with a 60 -min exposure time. This means that we can precisely measure radial velocities for late-type stars down to magnitude of about 11.5 mag. The spectral range covers 57 echelle orders with wavelength from $4280 \AA$ to $7500 \AA$.

The PST 2 is to be placed at least 120 degrees apart from the PST 1 in longitude. Both telescopes will form the GATS system which will allow for a high duty-cycle, nearly continuous monitoring of selected objects (see Fig. 1).

The GATS will be used to do research in the following topics:

- asteroseismology of hybrid pulsating stars, 


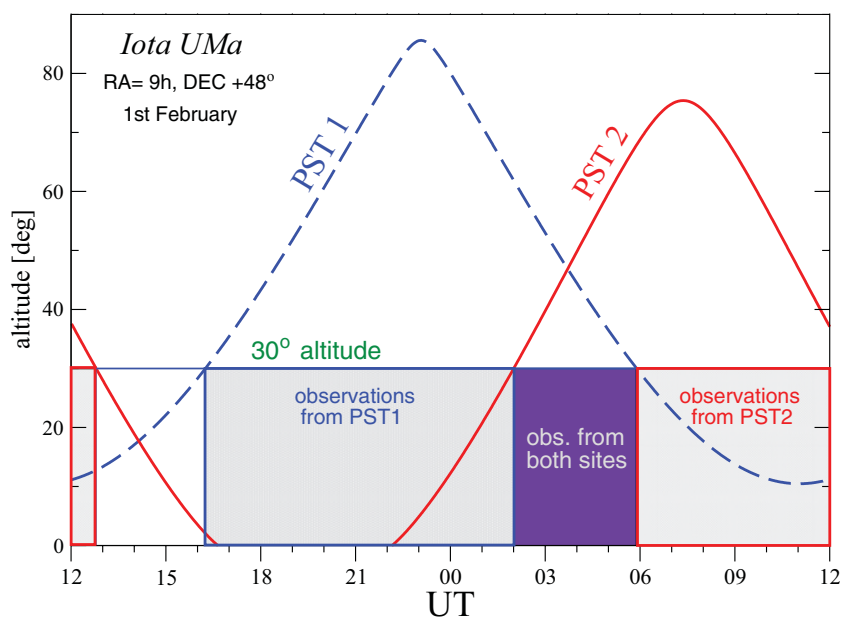

Figure 1. Changes of altitude of $\iota$ UMa as observed by the PST 1 (dashed line) and PST 2 (solid line), computed for 1st of February. The dark rectangle indicates the time when the object can be observed by both telescopes.

- stellar rotation and dynamical evolution of binary eclipsing stars,

- stellar activity cycles.

For more information, please visit the website of the GATS project: http://www.astro.amu.edu.pl/GATS.

\section{Reference}

Baranowski, R., Smolec, R., Dimitrov, W., et al. 2009, MNRAS, 396, 2194 